\title{
Optimal design of experiments applied to headspace solid phase microextraction for the quantification of vicinal diketones in beer through gas chromatography-mass spectrometric detection
}

\author{
João M. Leça ${ }^{\text {a, b }}$, Ana C. Pereira ${ }^{\text {a, c, * }}$, Ana C. Vieira ${ }^{\text {a }}$, Marco S. Reis ${ }^{c}$, José C. Marques ${ }^{\text {a }}$ \\ ${ }^{a}$ Centre of Exact Sciences and Engineering, University of Madeira, Campus da Penteada, 9000-390 Funchal, Portugal \\ ${ }^{\mathrm{b}}$ Institute of Nanostructures, Nanomodelling and Nanofabrication (I3N), University of Aveiro, Aveiro, Portugal \\ ${ }^{\text {c } C I E P Q P F}$, Department of Chemical Engineering, University of Coimbra, Pólo II - Rua Sílvio Lima, 3030-790 Coimbra, Portugal
}

\section{H I G H L I G H T S}

- Optimization of HS-SPME methodology using optimal design of experiments.

- In-depth study of extraction parameters and analysis of their optimal levels.

- Good results regarding linearity, sensitivity, selectivity, precision and accuracy.

- Automated, easy-to-operate and fast analytical methodology to control VKDs in beer.

\section{A R T I C L E I N F O}

Article history:

Received 2 April 2015

Received in revised form

6 June 2015

Accepted 12 June 2015

Available online 10 August 2015

\section{Keywords:}

Vicinal diketones

Headspace solid phase microextraction

Optimization

Optimal design of experiments
G R A P H I C A L A B S T R A C T

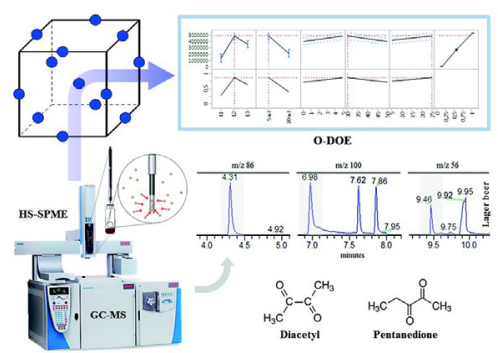

\begin{abstract}
A B S T R A C T
Vicinal diketones, namely diacetyl (DC) and pentanedione (PN), are compounds naturally found in beer that play a key role in the definition of its aroma. In lager beer, they are responsible for off-flavors (buttery flavor) and therefore their presence and quantification is of paramount importance to beer producers. Aiming at developing an accurate quantitative monitoring scheme to follow these off-flavor compounds during beer production and in the final product, the head space solid-phase microextraction (HS-SPME) analytical procedure was tuned through experiments planned in an optimal way and the final settings were fully validated. Optimal design of experiments (O-DOE) is a computational, statisticallyoriented approach for designing experiences that are most informative according to a well-defined criterion. This methodology was applied for HS-SPME optimization, leading to the following optimal extraction conditions for the quantification of VDK: use a CAR/PDMS fiber, $5 \mathrm{ml}$ of samples in $20 \mathrm{ml}$ vial, $5 \mathrm{~min}$ of pre-incubation time followed by $25 \mathrm{~min}$ of extraction at $30^{\circ} \mathrm{C}$, with agitation. The validation of the final analytical methodology was performed using a matrix-matched calibration, in order to minimize matrix effects. The following key features were obtained: linearity $\left(R^{2}>0.999\right.$, both for diacetyl and 2,3-pentanedione), high sensitivity (LOD of $0.92 \mu \mathrm{g} \mathrm{L}^{-1}$ and $2.80 \mu \mathrm{g} \mathrm{L}^{-1}$, and LOQ of $3.30 \mu \mathrm{g} \mathrm{L}^{-1}$ and $10.01 \mu \mathrm{g} \mathrm{L}^{-1}$, for diacetyl and 2,3-pentanedione, respectively), recoveries of approximately $100 \%$ and suitable precision (repeatability and reproducibility lower than $3 \%$ and $7.5 \%$, respectively). The
\end{abstract}

Abbreviations: VKDs, vicinal diketones; O-DOE, optimal design of experiments; HS-SPME, head space solid-phase microextraction; DC, diacetyl; PN, pentanedione; LG, lager beer.

* Corresponding author. Department of Chemical Engineering, University of Coimbra, Pólo II - Rua Sílvio Lima, 3030-790 Coimbra, Portugal.

E-mail addresses: acpereira@uma.pt, apereira@eq.uc.pt (A.C. Pereira). 
applicability of the methodology was fully confirmed through an independent analysis of several beer samples, with analyte concentrations ranging from 4 to $200 \mathrm{~g} \mathrm{~L}^{-1}$.

(C) 2015 Elsevier B.V. All rights reserved.

\section{Introduction}

The implementation of virtually any analytical procedure requires the specification of a set of experimental conditions from which its ultimate performance critically depends. Examples include the optimization of extraction parameters (e.g., temperature, time, pressure, sorbent) to ensure an adequate sample preparation, or of chromatographic settings (e.g., mobile phase composition, gradient parameters, $\mathrm{pH}$, temperature, injection volume and flow rate) in order to maximize the separation ability and quantification of the compounds of interest. Specifying all these different settings is a far from trivial task. It involves the simultaneous consideration of several factors (typically 5-10), some of them are easy to manipulate while others require demanding and labor intensive experimental procedures. Moreover, factors can be both quantitative (e.g., operation temperatures, extraction times, etc.) or qualitative (type of solvent, filter or coatings), having associated linear, bilinear or non-linear effects or including combinations that may not even be feasible or recommendable. In these circumstances, statistical design of experiments constitute a body of knowledge that can effectively assist the efforts of the analyst interested in taking the most out of the equipment available and in optimizing the entire experimental process [1-5]. Regarding the operational cost, statistical design of experiments (DOE) assumes clear advantages: the lowest cost and experimental overhead, since it implies lower number of trials and consequently, the consumption of reagents, standards and samples is reduced, as well as the time of analysis. Also, O-DOE guarantees statistically meaningful conclusions which are therefore scientifically valid, safeguards against erroneous and biased procedures leading to wrong or suboptimal conclusions which can occur when experiments are poorly planned. The latter scenario refers to univariate optimization, oneat-a-time manipulation of factors, non-random allocation of conditions or deficient consideration of experimental blocks. One-at-atime optimization, in particular, is one of the most recurrent approaches [6-10] often leading to suboptimal results as it does not even considers the existence of interactions between the factors under analysis. Most of the advantages of DOE in relation to such univariate strategy can be found in Leardi review [1].

Factorial designs are among the most popular methods for designing experiments, as they are straightforward to implement and provide results that can be very easily interpreted [11]. To analyze a high number of factors, the fractional factorial design is usually a good solution. These approaches have been widely applied in SPME optimization, when some extraction parameters are set or known a priori $[12,13]$. Fractional factorials are also used as a first step for multivariate optimization, where factors are screened in order to identify those with significant effects in the response variables and to investigate the nature of interactions between them [14]. Response surface designs (RSM) are the usual advanced choice to complete and refine the previous models in order to locate the optimum. More details about this class of designs can be found in the review papers of Bezerra et al. and Hanrahan et al. $[15,16]$. Several papers reporting additional technical information and applications examples of Central Composite designs [17,18], Box-Behnken design [19] and Doehlert matrix [20] are also available.
As illustrated above, the application of classical DOE methodologies to analytical chemistry has already been explored and demonstrated its potential in several relevant applications. Ferreira et al. [21] reviews the application of these methodologies to optimize extraction procedures and for determining the experimental conditions for chromatographic separations (optimization of mobile phases). Candioti et al. also review the most recent analytical applications of DOE methodologies when several responses have to be simultaneously optimized [22]. In the particular case of SPME optimization (the scope of this article) there are already some published studies, namely for quantifying furanic compounds on coated deep-fried products [23], to quantify trace quantities of phthalates in beer [24] and to analyze volatile compounds on cooked beef [25].

More recently, a new category of experimental designs has been growing in importance given its higher flexibility and optimal nature: the so-called optimal design of experiments (O-DOE). Its advantages include handling experimental regions that are not straightforward or even impossible to carry out. For example, it can be useful to limit the time involved in sample preparation, sidestep a scenario which require a number of resources or leads to high analysis costs, exclude a priori experimental settings that are not feasible due to technical limitations, or incorporate constrains on the total number of experiments in order to ensure that the sample volume and optimization costs are practicable. Furthermore, after each round of experiments, $\mathrm{O}-\mathrm{DOE}$ is also able to incorporate all the experimental information previously collected in the algorithm and compute the new experimental points to be tested that refine the knowledge acquired of the system in an optimal way, in some welldefined sense properly defined a priory. Several optimality criteria can be adopted for this purpose, such as those known as D-, A-, E-, G- and I-optimality [26]. Several applications related to sample preparation have been published which discuss and demonstrate the added-value of adopting O-DOE [27]. Hoogerbrugge et al. (2003) [28] used a D-optimal design to study and optimize five Supercritical Fluid Extraction parameters on polycyclic aromatic hydrocarbons extraction from earthworms. An experimental design based on the D-optimality criterion was also used by I. García [29] to evaluate the effect of five factors related to the extraction ciprofloxacin and enrofloxacin from eggs, in order to ensure high recoveries with small standard deviations. Stuart $\mathrm{H}$. Munson-McGee, in 2014, compared D- and G-optimal designs for the freeze concentration process [27].

In this paper, we focus on vicinal diketones (VDK), which are critical odor ketones in many fermented food and beverages, namely on beer. In particular, we will address the quantification of the following VDKs: DC, also known as butane-2,3-dione, and PN, commonly named 2,3-pentanedione. VDKs are produced by yeast during alcoholic fermentation [30-32]. DC and PN are formed by the oxidative decarboxylation reaction of $\alpha$-acetolactate and $\alpha$ acetohydroxybutyrate, respectively. In yeast cells, these VDKs result from the valine and isoleucine biosynthetic pathways and are then excreted to the extracellular medium. At the end of the fermentation and maturation phase, DC and PN are re-assimilated by yeast and reduced to acetoin and then to 2,3-butanediol. The latter compounds have higher odor thresholds when compared with DC, and therefore they are not so easily detected by the consumer 
These reactions are carried out through various enzymatic systems in the brewing yeast [33-35]. The DC and PN beer flavors are responsible for the main off-flavors and are usually used as target analytes to control the beer quality $[30,36]$. Therefore, their quantification is highly relevant for Quality Control purposes in the beer industry, a sector with significant economic impact in several regions of Europe.

DC is more "butterscotch" odor-active when compared to PN, and, in lager beers DC has an odor threshold around $100-200 \mu \mathrm{g} \mathrm{L}^{-1}$ while PN is around 900-1000 $\mu \mathrm{g} \mathrm{L}^{-1}[35,37]$. These compounds have attracted the attention of the scientific community, in particular DC which has been more thoroughly studied. Two types of approaches are reported in the technical literature to quantify DC. In the first approach, DC is directly analyzed like any other chemical volatile. On the other hand, in the second approach DC is transformed into a more stable derivative, due to its high reactivity and water solubility, which is then the target analyte of the methodology [35]. High performance liquid chromatography (HPLC), always with a derivatization step, has been used to determine, simultaneously, DC and PN in beer. However, gas chromatography coupled with mass spectrometry detection (GC-MS) presents higher levels of sensitivity for DC and PN quantification [38-42]. The concentration ratio, DC/PN, has also been used to access the microbe contamination degree during fermentation $[6,30,35]$. Regarding extraction techniques, the head space (HS), solid phase extraction (SPE) and SPME are the most commonly reported techniques for analysis the VDKs $[6,40,42,43]$. The free solvent HS-SPME technique has been successfully used in the simultaneous determination of DC and PN in different food matrices [44-46], including beer [47]. However, to the best of our knowledge, hitherto no HS-SPME methodology was optimized using state of the art O-DOE for quantification of both VKDs. This work presents for the first time the application of D-optimal design of experiments to optimize six different HS-SPME parameters (qualitative and quantitative), with limited experimental runs, to quantify VDKs in beer using GC-MS. The validated analytical procedure is simple to implement, easy-to-operate and fast, advantages called for quality control practices. SPME has the additional advantage of being a method that can be quickly tested and validated on the autosampler SPME mode, once its optimal extraction parameters are established.

\section{Materials and methods}

The workflow followed to optimize HS-SPME performance is summarized in Fig. 1. More details in each of the stages are provided in the following sections.

\subsection{Chemicals and samples}

DC and PN were purchased from Acros Organics (Geel, Belgium) and 4-methyl-2-pentanol (MP), used as internal standard (IS), was obtained from Sigma-Aldrich (Steinheim, Germany). All standards had a purity grade of more than $97 \%$, adequate to GC-MS analyses. Absolute ethanol, $>99.8 \%$ (GC), was purchased from Sigma-Aldrich (Steinheim, Germany). Ultra-pure water (conductivity of $18 \mathrm{M} \Omega$ ) was obtained by the Simplicity ${ }^{\mathbb{R}} \mathrm{UV}$ ultrapure water (type 1) apparatus from Millipore (Milford, MA, USA).

DC and PN stock solutions of $1 \mathrm{~g} \mathrm{~L}^{-1}$ were prepared in ultra-pure water. Then, suitable dilutions of the stock solutions were prepared with ultra-pure water, to obtain the intermediate solutions of $20 \mathrm{mg} \mathrm{L}^{-1}$ of DC, PN and MP. In order to obtain the matrix-matched calibration solution, the intermediate solutions were used to spike selected beer. Each calibration point was extracted in triplicate within the validation range $10-300 \mu \mathrm{g} \mathrm{L}^{-1}$.

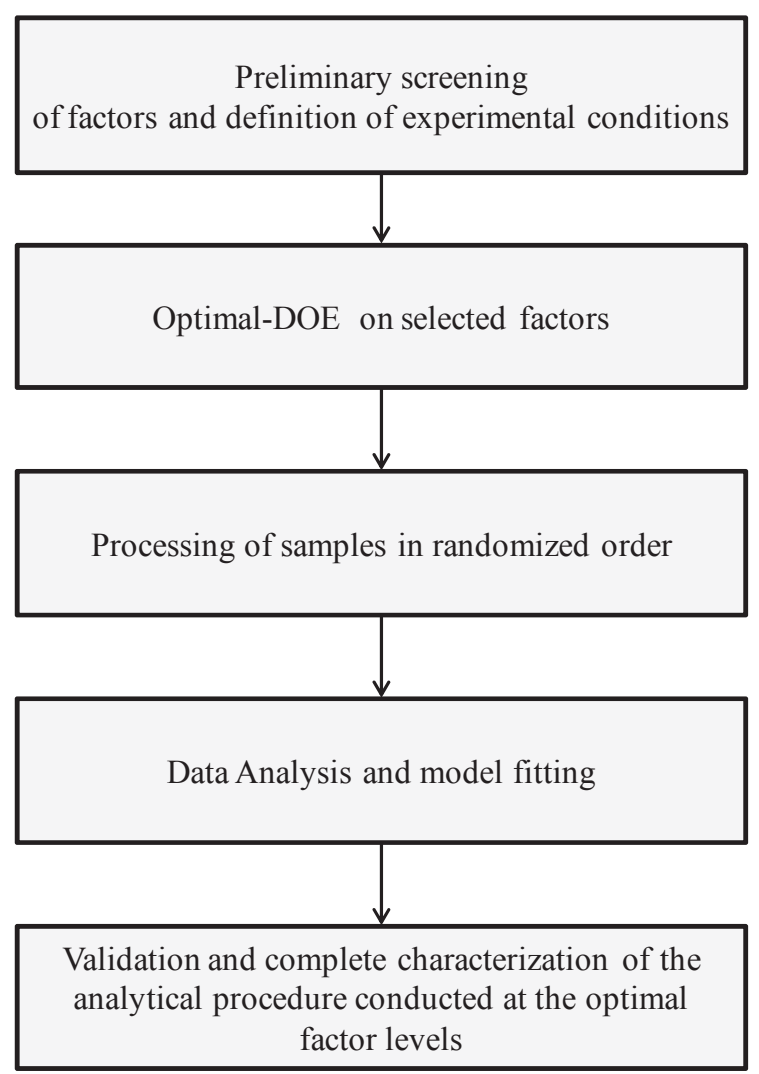

Fig. 1. The global analysis workflow for optimizing HS-SPME performance.

The lager beer used to perform the matrix-matched calibration was obtained from a local brewery, absent of quantifiable amounts of DC and PN. In the stage of application of the developed methodology, a sample set of 16 commercial beers were analyzed.

\subsection{Extraction apparatus and chromatographic conditions}

\subsubsection{HS-SPME materials and apparatus}

The SPME fibers tested were purchased from Supelco (Bellefonte, PA, USA): i) a stableflex fiber core coated with 50/30 $\mu \mathrm{m}$ divinylbenzene-carboxen-polydimethylsiloxane (DVB/Car/PDMS), ii) $85 \mu \mathrm{m}$ carboxen-polydimethylsiloxane (Car/PDMS) and iii) divinylbenzene-polydimethylsiloxane (DVB/PDMS), iv) fused silica core coated with $85 \mu \mathrm{m}$ polyacrylate (PA) and v) metal alloy fiber core coated with polyethylene glycol (PEG). All SPME tests were carried out on TriPlus autosampler, in SPME mode.

\subsubsection{Quantification procedure}

All analyses to identify and quantify VDK were carried out using a GC-MS system, the TRACE GC Ultra gas chromatograph coupled with an ISQ single quadrupole from Thermo Scientific (Hudson, NH, USA). We have employed a Factor Four capillary column, VF-5 ms $60 \mathrm{~m} \times 0.25 \mathrm{~mm}$ ID DF $=0.25$ (Varian, USA). The carrier gas was helium at a constant flow rate of $1 \mathrm{~mL} \mathrm{~min}^{-1}$. The injector port was kept at $240{ }^{\circ} \mathrm{C}$, in splitless mode, while the transfer line and the ion source were maintained at 230 and $240{ }^{\circ} \mathrm{C}$, respectively. The oven temperature program started at $40{ }^{\circ} \mathrm{C}$, hold for $3 \mathrm{~min}$, increased to $60{ }^{\circ} \mathrm{C}$ at $5^{\circ} \mathrm{C} \mathrm{min}^{-1}$, then increase again to $130{ }^{\circ} \mathrm{C}$, at $15^{\circ} \mathrm{C} \mathrm{min}-1$ and finally to $240{ }^{\circ} \mathrm{C}$, at $55^{\circ} \mathrm{C} \mathrm{min}^{-1}$, where it was held for $10 \mathrm{~min}$. The total GC run time was of $23.6 \mathrm{~min}$. This ramp of temperatures was tested and established after testing different concentrations of VDKs and IS in order to obtain the best resolution of the target 
compounds. The first chromatograms were obtained in full scan mode (total ion count) in order to obtain the retention times $\left(t_{R} s\right)$ of each target compound. After the confirmation of the $t_{R} S$, the analyses were always preformed with the characteristic and major ions of each analyte: $m / z 42,43$ and 86 for $\mathrm{DC}\left(\mathrm{t}_{\mathrm{R}}=4.30 \mathrm{~min}\right), \mathrm{m} / z 57$ and 100 for $\mathrm{PN}\left(\mathrm{t}_{\mathrm{R}}=6.97 \mathrm{~min}\right)$ and the ions $\mathrm{m} / z 41,43,56$ and 69 for MP $\left(t_{R}=9.40\right)$ as IS. Characteristic ions were used for quantification purposes, namely $m / z 86,100$ and 56 for DC, PN and MP, respectively, and also to build the calibration curves.

The mass spectrometer was operated in electron impact (EI) mode at $70 \mathrm{eV}$. The selective ion monitoring (SIM) operating mode was used with the characteristic ions for each target compound. From 1.8 to $5.5 \mathrm{~min}$ the ions $\mathrm{m} / \mathrm{z} 42,43$ and 86 were recorded, to analyze $D C\left(t_{R}=4.30 \mathrm{~min}\right)$; from 5.5 to $8.0 \mathrm{~min}$ the ions $\mathrm{m} / \mathrm{z} 57$ and 100 , to analyze $P N\left(t_{R}=6.97 \mathrm{~min}\right)$ and after $8.0 \mathrm{~min}$ the ions $\mathrm{m} / z 41$, 43,56 and 69 to analyze MP $\left(t_{R}=9.4\right)$ as IS.

\subsection{HS-SPME optimization}

After setting the basic experimental conditions as described in the previous sections, the factors to be contemplated in the optimization of the HS-SPME performance need to be defined (Step 1 in the workflow depicted in Fig. 1). After a careful consideration of the possibilities available, a preliminary factor screening was carried out following best judgment based on the accumulated background knowledge for this type of technique. This process converged to the set of factors or input variables presented in Table 1, where it is also shown the corresponding levels for qualitative (or categorical) factors and the minimum/maximum values that define the feasibility range for quantitative factors. In brief terms, we have considered the type of SPME fiber (DVB/Car/PDMS, Car/PDMS and DVB/PDMS), sample volume, pre-incubation time, extraction time, extraction temperature and agitation as input variables or factors, whereas the peak areas were selected as the response variable. As mentioned in Section 2.2.1, five types of fibers were initially considered, but PA and PEG were excluded after a preliminary step in which we identify matrix coelution problems, to be precise with DC. PDMS was also left out of the analysis since the polarity of this fiber coating is not suitable for the extraction of polar analytes (as VDK) [48]. Finally, sample volume was treated as a qualitative variable, as only two volumes are recommended to be used in the experimental apparatus.

The number of experiments (36) is a result of the trade-off between the material and time resources available and the accuracy needed to build the final model and estimate the optimal experimental conditions (Table 2). In this process we have considered a viable range for the number of experiments that can be accommodated by the available resources and compared the added-value of including an increasing number of experiments in the final accuracy of the models by looking to diagnostic tools such as the relative variance of the coefficients and the associated power, as well as the prediction variance surfaces. In order to extract the

Table 1

Experimental domain explored to optimize the HS-SPME extraction performance.

\begin{tabular}{lll}
\hline Factor & Qualitative/Quantitative & Levels \\
\hline Type of fiber & Qualitative & L1-DVB/PDMS, \\
& & L2-Car/PDMS, L3-DVB/ \\
& Car/PDMS \\
Sample volume & Qualitative & $\{5,10\}$ \\
Pre-incubation time $(\min )$ & Quantitative & {$[0,10]$} \\
Extraction time $(\min )$ & Quantitative & {$[5,25]$} \\
Extraction temperature $\left({ }^{\circ} \mathrm{C}\right)$ & Quantitative & {$[30,50]$} \\
Agitation & Qualitative & $\{$ L1-Yes, L2-No $\}$ \\
\hline
\end{tabular}

maximum amount of information that can be obtained using the available experimental resources, an optimal design of experiments (O-DOE) approach was followed to define the testing conditions (or treatments, in the usual DOE language) based on the D-optimality criterion. With this criterion, experimental points are allocated in the feasible region in an optimal way, with optimality defined in the sense of minimizing the determinant of the variance-covariance matrix of the inputs or, equivalently, maximizing the determinant of the information matrix for the linear regression model to be estimated. For a regression model defined in matrix terms as,

$\mathbf{Y}=\mathbf{F} \beta+\boldsymbol{\varepsilon}$

where, $\mathbf{Y}$ is the column with the response values, $\mathbf{F}$ the matrix of inputs and possibly their transformations (also known as the extended design matrix), $\beta$ the vector with the regression coefficients and $\varepsilon$ the vector of residuals, the corresponding information matrix is given by defined by $\mathbf{F}^{T} \mathbf{F}$. Therefore, D-optimality is defined in the terms of the following optimization problem:

$\operatorname{Max}_{\mathbf{F}} \phi_{D}=\left|\mathbf{F}^{T} \mathbf{F}\right|$

where, $|\cdot|$ is the determinant and ${ }^{T}$ stands for the matrix transpose operation. This criterion minimizes a functional of the variance of the model coefficient estimates or, in other words, of their associated uncertainty. As the variance-covariance matrix of the coefficients estimates is given by $\operatorname{Cov}(\boldsymbol{\beta})=\sigma_{\boldsymbol{\varepsilon}}^{2}\left(\mathbf{F}^{T} \mathbf{F}\right)^{-1}$ (where $\sigma_{\boldsymbol{\varepsilon}}^{2}$ is the variance of the residuals), minimizing the determinant of $\left(\mathbf{F}^{T} \mathbf{F}\right)^{-1}$, amounts to minimize the volume (or "size") of the inverse of the information matrix, leading to low variance estimates for the regression coefficients as well as low off-diagonal covariance terms, a desirable feature in regression models (since in this case the analysis of the model terms can be made individually). As the goals of the present work concern the identification of the key factors contributing to the HS-SPME performance and the maximization of response value, the accuracy of the model to be estimated from the experimental points is a key aspect, which justifies the choice of the D-optimality criterion $[26,49]$.

With the resources available to complete 36 different experimental points, the design should allow for the estimation of the main effects and second order interactions for all the 5 factors considered. The computational implementation of the D-optimal design is of an iterative nature and is conducted through the so called coordinate exchange algorithm [50]. At each iteration, this algorithm tests every value of every factor in the design to evaluate if changing that value would improve the optimality criterion. If so, the new value replaces the old one. This process continues until no replacement is made for a complete iteration. This is repeated several times using random initial conditions, in order to mitigate the possibilities of convergence to local optima.

The D-optimal design obtained by the coordinate exchange algorithm was preliminary assessed before implementing the sequence of experiments in random order. In order to verify that the design found is indeed suitable for the purposes of this study, we have analyzed the correlations between all model terms to be estimated, in order to detect if the limited number of samples is imposing some undesirable associations that ultimately lead to ambiguous parameters estimates. In Fig. 2 we present a color map for the correlation matrix of parameters estimates, where the magnitude of the correlation coefficients between the terms for all pair-wise combinations of factors is presented in a color scale. As can be easily verified, the amount of correlation in the off-diagonal terms is very low or residual, meaning that the proposed design is 
Table 2

Experimental design for HS-SPME optimization.

\begin{tabular}{|c|c|c|c|c|c|c|c|}
\hline Run & Fiber coating & $\begin{array}{l}\text { Incubation } \\
\text { time (T1), min }\end{array}$ & $\begin{array}{l}\text { Extraction } \\
\text { temperature, }{ }^{\circ} \mathrm{C}\end{array}$ & $\begin{array}{l}\text { Extraction time } \\
\text { (T2), min }\end{array}$ & Agitation & $\begin{array}{l}\text { Sample } \\
\text { volume (ml) }\end{array}$ & $\begin{array}{l}\text { Sum of peak areas } \\
(\mathrm{DC}+\mathrm{PN} 100 \mathrm{ppb})\end{array}$ \\
\hline 1 & DVBCarPDMS & 0 & 50 & 25 & $\mathrm{~L} 2$ & 5 & $40,48,187.0$ \\
\hline 2 & CarPDMS & 0 & 50 & 25 & L1 & 5 & $49,78,667.0$ \\
\hline 3 & DVBPDMS & 5 & 50 & 25 & $\mathrm{~L} 2$ & 10 & $505,980.0$ \\
\hline 4 & DVBPDMS & 5 & 30 & 25 & $\mathrm{~L} 1$ & 10 & $633,168.0$ \\
\hline 5 & DVBCarPDMS & 5 & 50 & 5 & L2 & 5 & $32,85,822.0$ \\
\hline 6 & CarPDMS & 0 & 50 & 5 & $\mathrm{~L} 2$ & 10 & $39,82,329.0$ \\
\hline 7 & DVBCarPDMS & 5 & 30 & 25 & $\mathrm{~L} 2$ & 10 & $37,71,416.0$ \\
\hline 8 & CarPDMS & 5 & 30 & 25 & L1 & 5 & $51,91,046.0$ \\
\hline 9 & DVBPDMS & 0 & 30 & 5 & $\mathrm{~L} 2$ & 10 & $650,956.0$ \\
\hline 10 & CarPDMS & 5 & 50 & 5 & L1 & 5 & $35,96,601.0$ \\
\hline 11 & DVBPDMS & 0 & 50 & 25 & L1 & 10 & $521,750.0$ \\
\hline 12 & DVBCarPDMS & 5 & 30 & 25 & L2 & 5 & $37,75,237.0$ \\
\hline 13 & DVBCarPDMS & 0 & 50 & 25 & $\mathrm{~L} 2$ & 10 & $31,25,246.0$ \\
\hline 14 & CarPDMS & 2.5 & 40 & 15 & $\mathrm{~L} 2$ & 5 & $46,47,051.0$ \\
\hline 15 & CarPDMS & 5 & 30 & 5 & $\mathrm{~L} 2$ & 10 & $27,91,309.0$ \\
\hline 16 & DVBPDMS & 0 & 30 & 25 & $\mathrm{~L} 2$ & 5 & $546,523.0$ \\
\hline 17 & CarPDMS & 0 & 30 & 5 & $\mathrm{~L} 2$ & 5 & $26,94,187.0$ \\
\hline 18 & CarPDMS & 5 & 50 & 25 & $\mathrm{~L} 2$ & 5 & $41,73,559.0$ \\
\hline 19 & DVBCarPDMS & 2.5 & 40 & 15 & L1 & 10 & $27,44,905.0$ \\
\hline 20 & DVBPDMS & 0 & 50 & 5 & L2 & 5 & $447,964.0$ \\
\hline 21 & DVBPDMS & 5 & 50 & 25 & L1 & 5 & $586,391.0$ \\
\hline 22 & CarPDMS & 0 & 30 & 25 & $\mathrm{~L} 2$ & 10 & $244,883.0$ \\
\hline 23 & DVBCarPDMS & 5 & 30 & 5 & L1 & 5 & $24,20,638.0$ \\
\hline 24 & DVBCarPDMS & 0 & 30 & 25 & L1 & 5 & $27,72,221.0$ \\
\hline 25 & DVBCarPDMS & 0 & 50 & 5 & L1 & 5 & $23,60,300.0$ \\
\hline 26 & DVBCarPDMS & 5 & 50 & 5 & L2 & 10 & $24,74,674.0$ \\
\hline 27 & DVBCarPDMS & 0 & 30 & 5 & $\mathrm{~L} 2$ & 10 & $23,32,587.0$ \\
\hline 28 & DVBPDMS & 5 & 30 & 5 & $\mathrm{~L} 2$ & 5 & $656,437.0$ \\
\hline 29 & DVBPDMS & 5 & 50 & 25 & L2 & 10 & $705,800.0$ \\
\hline 30 & DVBCarPDMS & 5 & 30 & 5 & L1 & 10 & $23,20,368.0$ \\
\hline 31 & CarPDMS & 0 & 30 & 5 & L1 & 10 & $20,65,877.0$ \\
\hline 32 & CarPDMS & 5 & 50 & 25 & L1 & 10 & $13,08,630.0$ \\
\hline 33 & DVBCarPDMS & 5 & 50 & 25 & L1 & 5 & $21,90,679.0$ \\
\hline 34 & CarPDMS & 5 & 50 & 5 & L1 & 5 & $31,51,663.0$ \\
\hline 35 & DVBPDMS & 0 & 30 & 5 & L1 & 5 & $488,405.0$ \\
\hline 36 & DVBPDMS & 5 & 50 & 5 & L1 & 10 & $569,784.0$ \\
\hline
\end{tabular}

indeed able to provide unambiguous estimates of the different model terms.

More details on the implementation of O-DOE will be provided in the results section, namely those regarding Step 4 in the workflow presented in Fig. 1. Optimal design computations and analysis were carried out in the JMP software, version 11.0.0 (SAS Institute Inc.)

\subsection{Validation of the analytical methodology}

The optimal experimental condition (i.e., the optimal treatment, as a result of the O-DOE study) for implementing HS-SPME/GC-MS for the simultaneous determination of DC and PN was fully validated in terms of linearity, sensitivity, matrix effects, selectivity, precision and accuracy (last step in the workflow of Fig. 1). A specially selected lager beer was spiked with DC, PN and MP in- the respective calibration curves. The $100 \mu \mathrm{g} \mathrm{L}^{-1}$ of MP was also added to all analyzed beer samples and the concentrations of each VDK (DC and PN) was extrapolated by area ratio (analyte/IS).

The linearity $\left(\mathrm{R}^{2}\right)$, limit of detection (LOD) and limit of quantification (LOQ) were computed based on a linear regression approach, following the IUPAC recommendations [51]. The values obtained for these quantities were: $\mathrm{LOD}=3.3 \sigma / \mathrm{b}$ and $\mathrm{LOQ}=10 \sigma / b$, where $\sigma$ stands for the intercept standard deviation, and $b$ is the slope.

A matrix effect (ME) study was also carried out in order to verify the influence of beer matrix in the extraction of target compounds (DC and PN), when compared to a solution with $5.1 \%$ of ethanol. The slopes of calibration curves obtained by spiking the solution of $5.1 \%$ of ethanol and the beer (matrix-matched calibration), were compared by computing the following quantity [52]:

$\% \mathrm{ME}=\left[\frac{(\text { slope of matrix }- \text { matched calibration }- \text { slope of } 5.1 \% \text { of ethanol calibration })}{\text { slope of } 5.1 \% \text { of ethanol calibration }}\right] \times 100$

termediate solutions of $20 \mathrm{mg} \mathrm{L}^{-1}$ to prepare the 5 concentration range levels: $10,25,50,100$ and $300 \mu \mathrm{g} \mathrm{L}^{-1}$ of DC and PN with $100 \mu \mathrm{g} \mathrm{L}^{-1}$ of MP as IS. Each VDK compound area ratio (DC area/MP area; PN area/MP area), from all increasing standard solutions, was plotted against the corresponding concentration in order to obtain
Selectivity was confirmed by the absence of chromatographic interferences at the retention times of DC, PN and MP in the special selected lager beer, used for the matrix-matched calibration and several other commercial beers.

Inter- and intra-day analysis of 2 standard solutions, with 


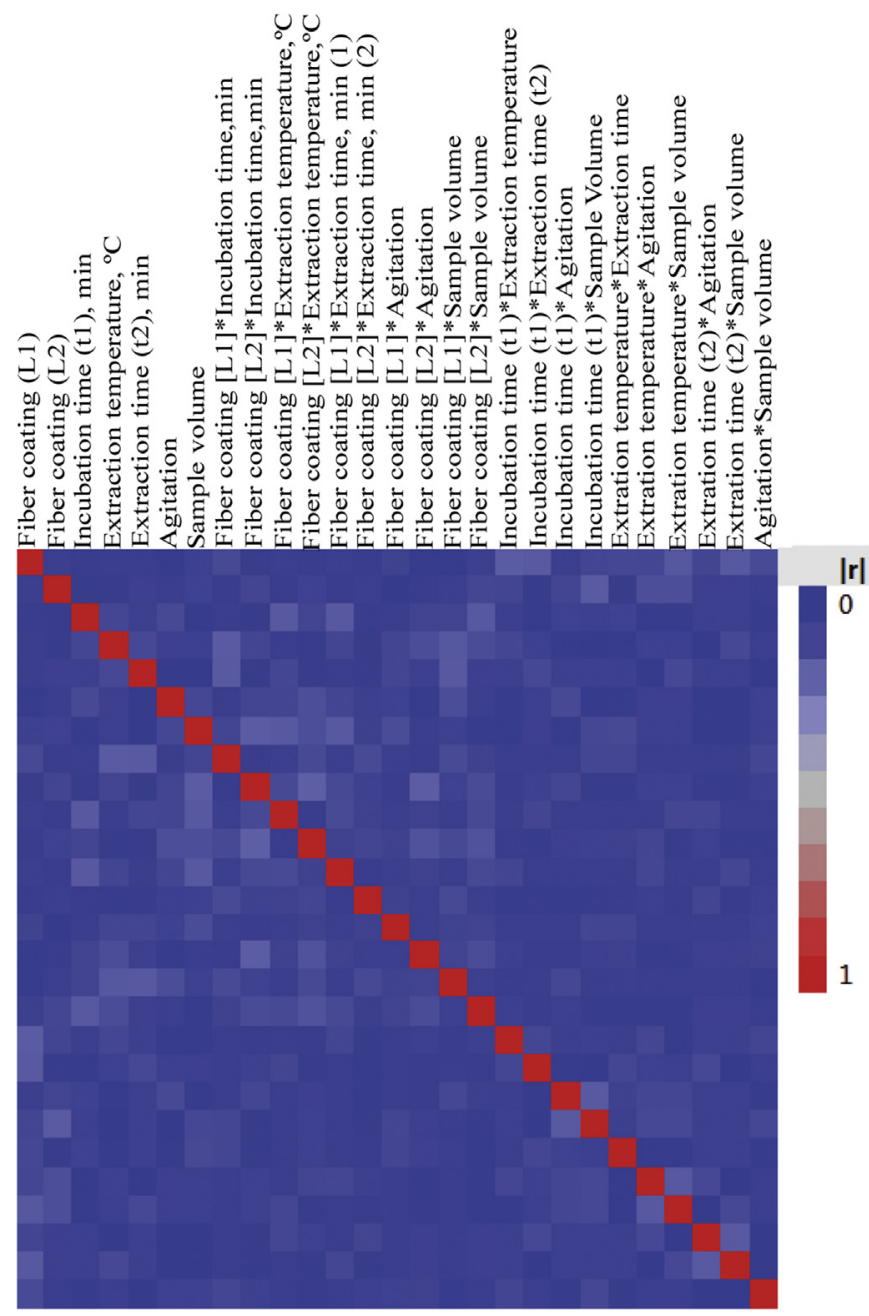

Fig. 2. Color map for the correlation matrix of parameters estimates. (For interpretation of the references to colour in this figure legend, the reader is referred to the web version of this article.)

different concentrations of DC and PN (100 and $200 \mu \mathrm{g} \mathrm{L}^{-1}$ ), was conducted in order to evaluate the method's precision. Repeatability (intra-day) was determined by the quantification of 7 successive replicates of each standard solution and reproducibility (inter-day) was assessed by performing the same analysis in 3 different days, in a period of over a week. The variations obtained in these parameters were expressed in terms of relative standard deviation (\%RSD).

A recovery study was carried out to determine the method's accuracy. A selected commercial beer was spiked, in triplicate, with known amounts of DC and PN covering 3 different concentrations levels of the calibration range $\left(25,100\right.$ and $\left.200 \mu \mathrm{g} \mathrm{L}^{-1}\right)$. The recovery was calculated according to the following expression:

Recovery $=\left[\frac{C_{m}-C_{0}}{C_{+}}\right] \times 100$

where $C_{m}$ is the measured VDK concentration in a spiked sample, $C_{0}$ is the measured concentration of VDK in the unspiked sample, and $C_{+}$is the concentration of VDK added to beer [53-55].

Carry-over was also tested by running a $5.1 \%$ of ethanol solution after the extraction of the highest concentrated working standard solution $\left(300 \mu \mathrm{g} \mathrm{L}^{-1}\right)$. This also allow us adjust the fiber conditioning time.

\section{Results and discussion}

In this section we address the fourth and fifth stages of the workflow presented in Fig. 1. These stages regard the results of the implementation of the D-optimal design and the subsequent complete characterization of the best experimental conditions found, as well as the validation of such optimal treatment and its application in the analysis of independent samples. In this context, the entire cycle of optimization is completed, from factors definition and elicitation to the characterization, validation and test of the final optimal solution.

\subsection{O-DOE results for HS-SPME extraction optimization}

After running the set of 36 experiments in random order, the values for the response variable (peak areas) were recorded and used to estimate the effects associated with all factors considered, as well as their interactions. The effects were estimated considering both analytes simultaneously, namely, the sum of both peak areas. The initial model contemplating all effects revealed that a large number of them are not indeed significant. This sparsity in factors relevance is quite typical in practice, and therefore the initial model should be revised in order to remove the terms not carrying any explanation power regarding the response variable. Fig. 3 presents the estimated scaled coefficients, where it is apparent the existence of a high portion of small-magnitude coefficients, being the most important effects related primarily with fiber coating and, to a lesser extent, sample volume.

Removing the terms corresponding to non-significant effects and re-estimating the model, one obtains the model presented in Table 3, where all parameters are now significant at a significance level of 0.01 (or even lower).

This model has an $R^{2}$ of 0.87 and an $R_{a d j}^{2}$ of 0.84 , indicating a good fit to the experimental data. By inspection of the estimated reduced model or by formally maximizing the desirability function for this design (related to the maximization of the separation performance), one finally obtains the desired optimal solution. The optimal operation condition for conducting HS-SPME extraction is depicted in Fig. 4 and summarized in Table 4.

As mentioned before, inspection of the final model reveals that the key factors with more influence in the response are the type of fiber coating and the sample volume. In Fig. 5 we present the nature of their influence with resort to a multi-vary chart. This chart stratifies the response by taking its average for the different levels and combination of levels of the factors involved, enabling an assessment of their influence either in an isolated or combined fashion. The fiber type CarPDMS is the one leading to highest average response level and also the one for which the sensitivity to changing conditions in sample volume is larger, being the best condition achieved for the volume of $5 \mathrm{ml}$.

With the optimal experimental conditions for conducting HSSPME extraction found, the final stage in the study workflow (Fig. 1 ) regards the complete characterization, validation and test of the proposed solution. These topics will be presented in detail in the next sections.

\subsection{Analytical procedure optimization and validation}

\subsubsection{Assessment of matrix effects}

Beer is a global consumption product characterized by a matrix of high complexity $[6,32,35,36]$. When analyzing this type of products, the matrix effect on extraction and consequently on the response of the GC-MS equipment should be evaluated and taken into account [52]. In this sense and assuming that the matrix effect can compromise the results generated by the proposed analytical 


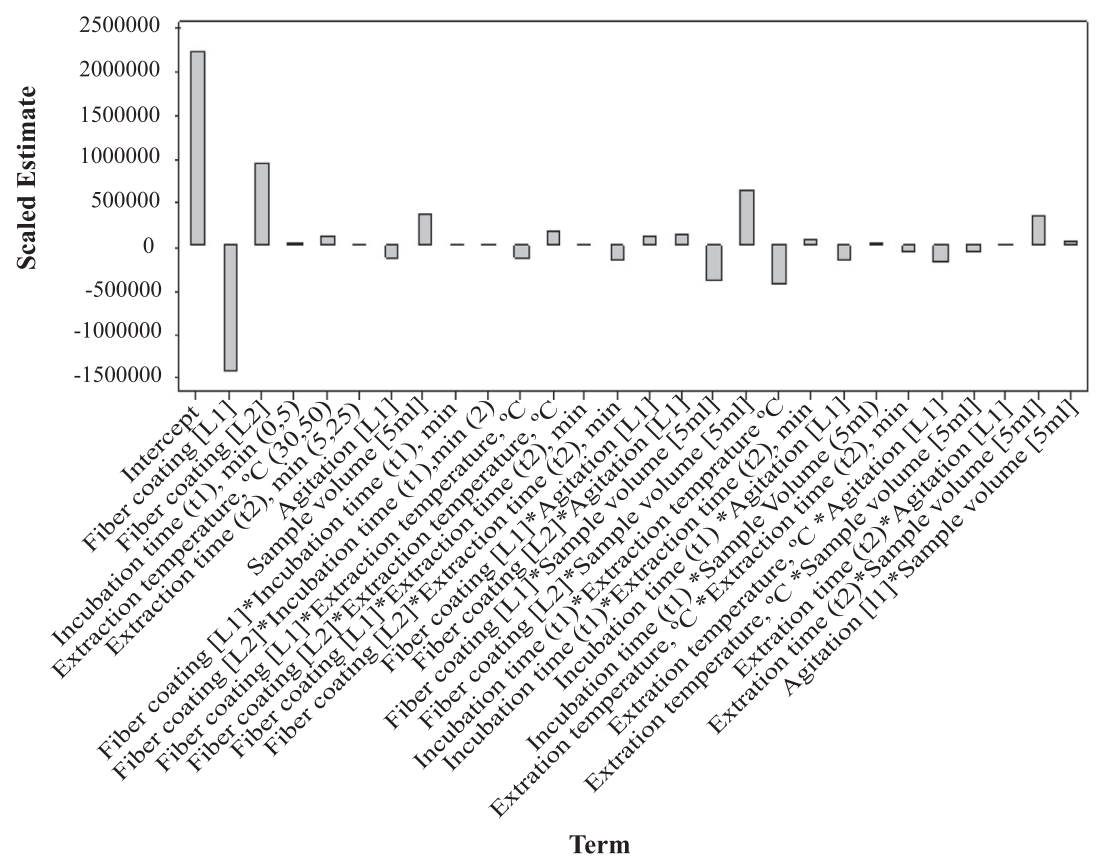

Fig. 3. Scaled estimates for the coefficients of the initial model containing all factors (including those that are not statistically significant).

Table 3

Summary of the model results after removing the non-significant terms from the initial model.

\begin{tabular}{|c|c|c|c|c|}
\hline Term & Estimate & Std error & t Ratio & p-Value \\
\hline Intercept & $22,28,533$ & $99,982.21$ & 22.29 & $<.0001$ \\
\hline Fiber coating [L1] & $-14,54,499$ & $146,721.3$ & -9.91 & $<.0001$ \\
\hline Fiber coating [L2] & $913,620.59$ & $141,551.6$ & 6.45 & $<.0001$ \\
\hline Sample volume $[5 \mathrm{ml}]$ & $355,669.69$ & $99,221.17$ & 3.58 & 0.0013 \\
\hline Fiber coating [L1] * sample volume [5 ml] & $-4,32,678$ & $143,497.8$ & -3.02 & 0.0054 \\
\hline Fiber coating [L2] * sample volume $[5 \mathrm{ml}]$ & $687,234.14$ & $141,018.1$ & 4.87 & $<.0001$ \\
\hline Incubation time (T1), min * Extraction temperature, ${ }^{\circ} \mathrm{C}$ & $-4,31,314$ & 104597.3 & -4.12 & 0.0003 \\
\hline Extraction time (T2), $\min ^{*}$ Sample volume $[5 \mathrm{ml}]$ & $328,093.19$ & $103,677.2$ & 3.16 & 0.0037 \\
\hline
\end{tabular}

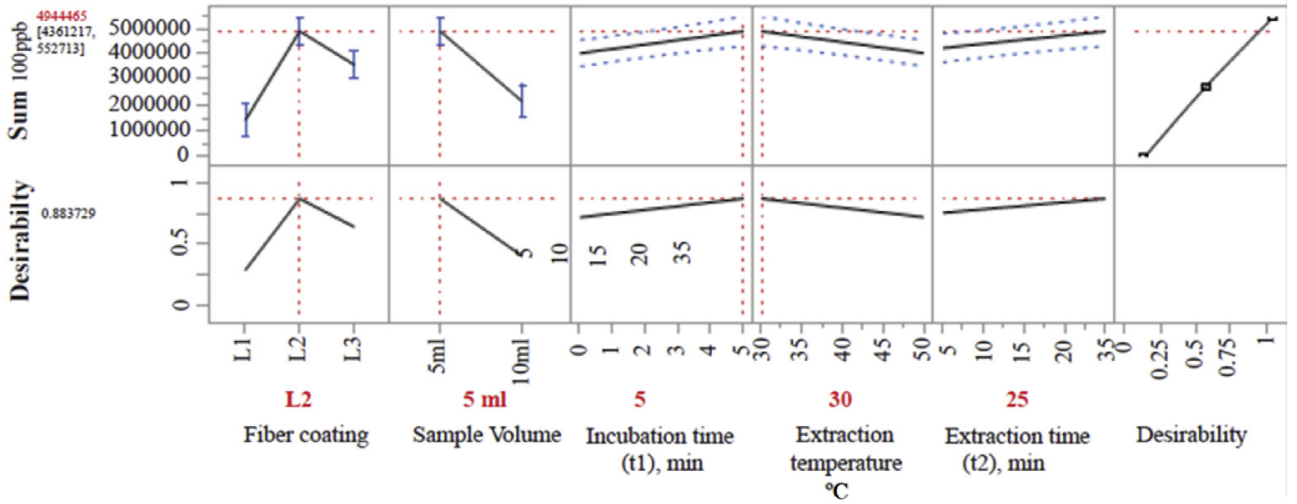

Fig. 4. Profiles for the predicted response as a function of the input factors, with an indication of the optimal levels obtained with the D-optimal design.

method, the variation percentages of the slopes of the calibration curves obtained for DN and PN, generated with $5.1 \%$ of ethanol and beer (used for the matrix-matched calibration), were compared in order to evaluate the matrix influence on the extraction procedure and analysis of DC and PN. A number of beer samples were analyzed prior to matrix effect analysis, in order to confirm the absence of interferences at diacetyl and pentanedione retention times.

Although there are no uniformed and established limit values for detecting a matrix effect, it can be considered that values of matrix effect suppression or enhancement lower than $15 \%$ are usually considered acceptable. In this specific case, the matrix effect 
Table 4

Optimal levels for the experimental factors optimizing HS-SPME performance.

\begin{tabular}{ll}
\hline Factor & Optimal level \\
\hline Type of fiber & L2-Car/PDMS \\
Sample volume & $5 \mathrm{ml}$ \\
Pre-incubation time $(\mathrm{min})$ & $5 \mathrm{~min}$ \\
Extraction time $(\mathrm{min})$ & $25 \mathrm{~min}$ \\
Extraction temperature $\left({ }^{\circ} \mathrm{C}\right)$ & $30{ }^{\circ} \mathrm{C}$ \\
Agitation & $\mathrm{L} 1-\mathrm{Yes}$ \\
\hline
\end{tabular}

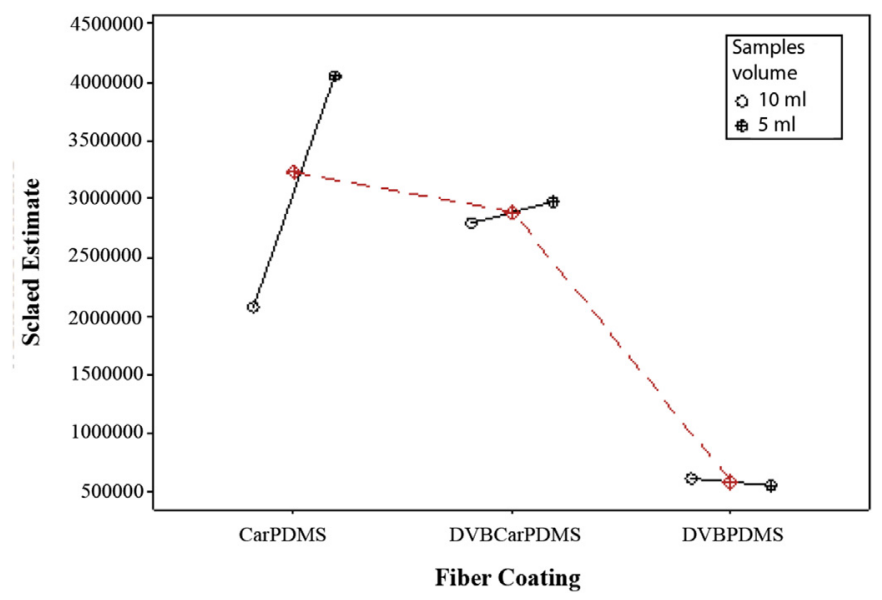

Fig. 5. Multi-vari chart for the influence of fiber coating and sample volume on the response variable.

between $5.1 \%$ of ethanol and the beer was $18.5 \%$ and $10.5 \%$ for DC and PN, respectively. Therefore, matrix which initially was intended to use to perform the calibration, 5.1\% of ethanol, revealed to be adequate for the quantification of PN (10.5\% of matrix effect) and less suitable for the DC analyses (18.5\%) of matrix effect. Table 5 summarizes the matrix effects obtained calculated according to equation (3) $[56,57]$. In this context, matrix effects must be considered in the analysis of future samples, which can be done through the adoption of a matrix-matched calibration approach for both DC and PN compounds.

\subsubsection{Method validation}

Faced with a higher matrix effect for DC (18.5\%), a matrixmatched calibration approach was adopted for both DC and PN compounds. The selectivity of the methodology was confirmed by the absence of interferents at DC and $\mathrm{PN} \mathrm{t}_{\mathrm{R}} \mathrm{S}$ ( $4.9 \mathrm{~min}$ and $6.9 \mathrm{~min}$, respectively) in the beer used to generate the matrix-matched calibration. Also, it was confirmed the absence of any coeluted interferents with target compounds, when commercial beer is used (Fig. 6).

LOD and LOQ were determined in order to confirm the sensitivity of the proposed methodology (as described in Section 2.3). The results obtained for LOD and LOQ were 0.92 and $2.80 \mu \mathrm{g} \mathrm{L}^{-1}$ to DC and 3.30 and $10.01 \mu \mathrm{g} \mathrm{L}^{-1}$ to PN (Table 6), being close or even lower to those found in literature $[6,30,38,40,42,47]$ and confirming

Table 5

Percentage of the matrix effect between the slopes of the three calibration curves: synthetic (5.1\% of ethanol) and commercial beer.

\begin{tabular}{lll}
\hline & DC & PN \\
\hline Synthetic vs commercial beer & $18.5 \%$ & $10.5 \%$ \\
\hline
\end{tabular}

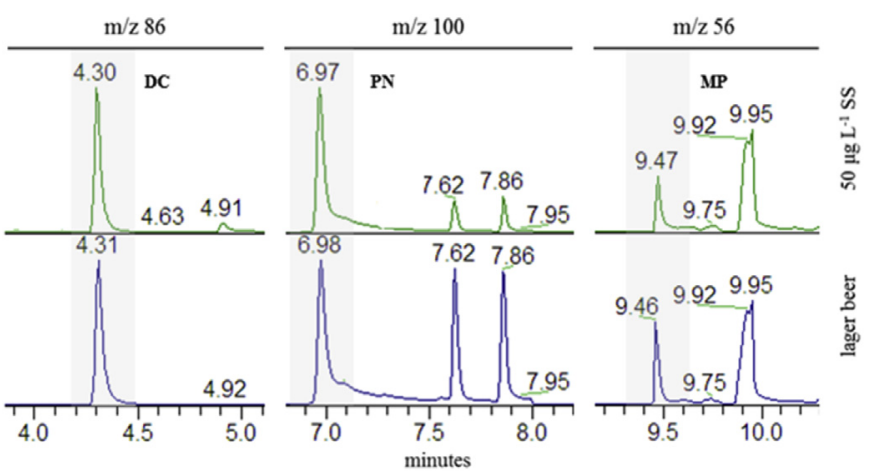

Fig. 6. Chromatograms of a $50 \mu \mathrm{g} \mathrm{L}^{-1}$ standard solution used to generate the matrixmatched calibration and an example of a lager beer sample. DC - diacetyl; PN pentanedione; MP - 4-methyl-2-pentanol; SS - standard solution.

Table 6

Validation results obtained for the HS-SPME/GC-MS methodology to quantify DC and PN.

\begin{tabular}{lll}
\hline Parameter & Diacetyl & Pentanedione \\
\hline Linear regression $(y=m x+b)$ & $0.0026 \mathrm{x}+0.0538$ & $0.0057 \mathrm{x}-0.0316$ \\
Linear concentration range & $10-300 \mu \mathrm{g} \mathrm{L}^{-1}$ & $10-200 \mu \mathrm{g} \mathrm{L}^{-1}$ \\
$\mathrm{R}^{2}$ & 0.9999 & 0.9997 \\
$\mathrm{LOD}\left(\mu \mathrm{g} \mathrm{L}^{-1}\right)$ & 0.9 & 3.3 \\
$\mathrm{LOQ}\left(\mu \mathrm{g} \mathrm{L}^{-1}\right)$ & 2.8 & 10.0 \\
Recovery & $\%$ & \\
$\mathrm{LB}+50 \mu \mathrm{g} \mathrm{L}^{-1} \mathrm{SA}$ & 91 & 102 \\
$\mathrm{LB}+100 \mu \mathrm{g} \mathrm{L}^{-1} \mathrm{SA}$ & 97 & 99 \\
$\mathrm{LB}+200 \mu \mathrm{g} \mathrm{L}^{-1} \mathrm{SA}$ & 94 & 91 \\
\hline
\end{tabular}

LOD - limit of detection; LOQ - limit of quantification; LB - lager bear; SD standard deviation; SA - Standard addition.

that the proposed methodology has an adequate sensibility to the intended determinations.

The method accuracy was assessed through a recovery study at 3 different concentration levels of the concentration range (Table 6). The commercial beer was analyzed in terms of DC and PN concentration, before and after the target analytes' fortification. The recoveries ranged between 91.0 and 101.6\%, confirming the method's accuracy.

The precision of the proposed methodology was evaluated in terms of repeatability and reproducibility by the variation of intraand inter-day analyses using two different working standard solutions ( 100 and $200 \mu \mathrm{g} \mathrm{L}^{-1}$ ). Good results were obtained in terms of repeatability and reproducibility, $2.0-2.7 \%$ and $1.1-2.0 \%$ in DC and $\mathrm{PN}$, respectively, for intra-day analyses and $2.9-7.3 \%$ and 2.9-7.4\% in DC and PN for inter-day analyses.

\subsection{Application to the analysis of new commercial beer samples}

To demonstrate the applicability and selectivity of the proposed HS-SPME methodology for the simultaneous determination of DC and PN in lager beer, a sample set of 11 randomly selected commercial beers and 5 aged beers was analyzed in order to cover a wide range of DC and PN concentration. All samples were extracted in triplicate and the concentration of DC and PN was extrapolated by the area ratio (VDK/IS) with the corresponding calibration curve. The obtained results are shown in Table 7. The chromatograms and the quantification results confirm that the methodology is selective (good peak resolution) and adequate for these VDKs quantification. The quantification results varied from 6.5 to $294 \mu \mathrm{g} \mathrm{L}^{-1}$ for DC and $15.4-141 \mu \mathrm{g} \mathrm{L}^{-1}$ for PC. 
Table 7

Application of the HS-SPME/GC-MS methodology to quantify and PN in 16 commercial and special beers.

\begin{tabular}{|c|c|c|c|c|}
\hline \multirow[t]{2}{*}{ Sample } & \multicolumn{2}{|l|}{ Diacetyl } & \multicolumn{2}{|c|}{ Pentanedione } \\
\hline & $\mathrm{Cc}\left(\mu \mathrm{g} \mathrm{L}^{-1}\right)$ & SD & $\mathrm{Cc}\left(\mu \mathrm{g} \mathrm{L}^{-1}\right)$ & SD \\
\hline $\mathrm{LB}_{1}$ & n.d. & - & n.q. & - \\
\hline $\mathrm{LB}_{2}$ & 40.7 & 2.4 & 17.0 & 0.6 \\
\hline $\mathrm{LB}_{3}$ & 207.8 & 3.3 & 27.7 & 0.2 \\
\hline $\mathrm{LB}_{4}$ & 294.9 & 21.4 & 30.6 & 3.5 \\
\hline $\mathrm{LB}_{5}$ & 34.5 & 0.7 & 20.6 & 1.0 \\
\hline $\mathrm{LB}_{6}$ & 83.8 & 3.2 & 23.8 & 0.1 \\
\hline $\mathrm{LB}_{7}$ & 61.6 & 3.6 & 17.6 & 0.6 \\
\hline $\mathrm{LB}_{8}$ & n.d. & - & 17.8 & 0.7 \\
\hline $\mathrm{LB}_{9}$ & 6.5 & 1.6 & 17.8 & 0.2 \\
\hline $\mathrm{LB}_{10}$ & n.d. & - & 15.4 & 0.7 \\
\hline $\mathrm{LB}_{11}$ & 34.7 & 0.6 & 52.9 & 3.0 \\
\hline $\mathrm{LB}_{12}$ & 74.9 & 4.0 & 76.4 & 3.0 \\
\hline $\mathrm{LB}_{13}$ & 118.7 & 1.4 & 141.0 & 3.9 \\
\hline $\mathrm{LB}_{14}$ & n.d. & - & 20.4 & 1.0 \\
\hline $\mathrm{LB}_{15}$ & n.d. & - & 18.3 & 0.4 \\
\hline $\mathrm{LB}_{16}$ & n.d. & - & 18.6 & 0.1 \\
\hline
\end{tabular}

LB - lager beer; Cc - Concentration; SD - standard deviation; n.q. - not quantified; n.d. - not detected.

\section{Conclusions}

Application of DOE methodologies in the development of analytical procedures and their optimization is currently a crucial step. This is particularly true for SPME procedures since there are several qualitative and quantitative parameters at stake and limited experimental resources. Therefore, experiments should be planned in an optimal way in order to extract as much relevant information as possible regarding the key drivers of variability, main interactions and eventually also non-linear effects that may be present. In this study, six SPME parameters where evaluated, namely fiber coating, sample volume, pre-incubation time, extraction time, extraction temperature and agitation. Fiber coating and sample volume were found to be the factors playing a major role in the extraction process. Several interaction effects also stand out, such as fiber coating $\times$ sample volume, incubation time $\times$ extraction temperature and extraction time $\times$ sample volume. According to the O-DOE results, the following conditions were established to quantify VDKs by HS-SPME priori to GCMS: fiber type CAR-PDMS, $5 \mathrm{~min}$ of pre-incubation followed by an extraction of $30 \mathrm{~min}$, always at $30{ }^{\circ} \mathrm{C}$, considering $5 \mathrm{ml}$ of sample in a $20 \mathrm{ml}$ vial with agitation.

Matrix effects were found be relevant in the present case (beer samples), and therefore a matrix-matched calibration approach was implemented. The proposed analytical methodology was fully characterized and validated, showing good results in terms of linearity, sensitivity, selectivity precision and accuracy. Its application to independent samples also demonstrates that it is selective (good peak resolution) and adequate for VDKs quantification.

With the application of O-DOE it was possible to optimize the HS-SPME extraction procedure within the constraints of available material and time resources, thus contributing to the implementation of faster, cheaper and more efficient procedures in the analytical laboratories. The interactions between all factors studied were analyzed, ensuring that the optimal conditions founded were correctly estimated.

\section{Acknowledgments}

The authors acknowledge Empresa de Cerveja da Madeira for kindly supplying the samples used in this work. João M. Leça and Ana C. Pereira acknowledge Agência Regional para o
Desenvolvimento da Investigação Tecnologia e Inovação (ARDITI) for the financial support through their grants, 0002376/2014/131 and 002248/2013/131, respectively.

The authors acknowledge the FEDER (Intervir + program) for the financial support of VALIMED project (MADFDR-01-0224FEDER-000006).

\section{References}

[1] R. Leardi, Experimental design in chemistry: a tutorial, Anal. Chim. Acta 652 (2009) $161-172$.

[2] D.B. Hibbert, Experimental design in chromatography: a tutorial review, J. Chromatogr. B 910 (2012) 2-13.

[3] M.P. Callao, Multivariate experimental design in environmental analysis, Trend Anal. Chem. 62 (2014) 86-92.

[4] G.E.P. Box, J.S. Hunter, W.G. Hunter, Statistics for Experimenters: Design, Innovation, and Discovery, second ed., Wiley, Hoboken, NJ (USA), 2005.

[5] D.C. Montegomery, Design and Analysis of Experiments, fourth ed., Wiley, New York, 1997.

[6] J. Tian, Determination of several flavours in beer with headspace sampling-gas chromatography, Food Chem. 123 (2010) 1318-1321.

[7] G. Charry-Parra, M. DeJesus-Echevarria, F.J. Perez, Beer volatile analysis: optimization of HS/SPME coupled to GC/MS/FID, J. Food Sci. 76 (2011) C205-C211.

[8] G.C.d. Silva, A.A.S.d. Silva, L.S.N.d. Silva, R.L.d.O. Godoy, L.C. Nogueira, S.L. Quitério, R.S.L. Raices, Method development by GC-ECD and HSSPME-GC-MS for beer volatile analysis, Food Chem. 167 (2015) 71-77.

[9] J.E. Welke, M. Zanus, M. Lazzarotto, C.A. Zini, Quantitative analysis of headspace volatile compounds using comprehensive two-dimensional gas chromatography and their contribution to the aroma of Chardonnay wine, Food Res. Int. 59 (2014) 85-99.

[10] J.L. Gonçalves, J.A. Figueira, F.P. Rodrigues, L.P. Ornelas, R.N. Branco, C.L. Silva, J.S. Câmara, A powerful methodological approach combining headspace solid phase microextraction, mass spectrometry and multivariate analysis for profiling the volatile metabolomic pattern of beer starting raw materials, Food Chem. 160 (2014) 266-280.

[11] J.M. Leça, V. Pereira, A.C. Pereira, J.C. Marques, Rapid and sensitive methodology for determination of ethyl carbamate in fortified wines using microextraction by packed sorbent and gas chromatography with mass spectrometric detection, Anal. Chim. Acta 811 (2014) 29-35.

[12] J.N. Bianchin, G. Nardini, J. Merib, A.N. Dias, E. Martendal, E. Carasek, Screening of volatile compounds in honey using a new sampling strategy combining multiple extraction temperatures in a single assay by HS-SPME-GC-MS, Food Chem. 145 (2014) 1061-1065.

[13] G. Fratini, S. Lois, M. Pazos, G. Parisi, I. Medina, Volatile profile of Atlantic shellfish species by HS-SPME GC/MS, Food Res. Int. 48 (2012) 856-865.

[14] J.N. Bianchin, G. Nardini, J. Merib, A.N. Dias, E. Martendal, E. Carasek, Determination of steroids, caffeine and methylparaben in water using solid phase microextraction-comprehensive two dimensional gas chromatography-time of flight mass spectrometry, Food Chem. 145 (2014) 1061-1065.

[15] G. Hanrahan, K. Lu, Application of factorial and response surface methodology in modern experimental design and optimization, Crit. Rev. Anal. Chem. 36 (2006) 141-151.

[16] M.A. Bezerra, R.E. Santelli, E.P. Oliveira, L.S. Villar, L.A. Escaleira, Response surface methodology (RSM) as a tool for optimization in analytical chemistry, Talanta 76 (2008) 965-977.

[17] T. Lundstedt, E. Seifert, L. Abramo, B. Thelin, Ä. Nyström, J. Pettersen, R. Bergman, Experimental design and optimization, Chemom. Intell. Lab. Syst. 42 (1998) 3-40.

[18] N. Moreira, S. Meireles, T. Brandão, P.G.d. Pinho, Optimization of the HSSPME-GC-IT/MS method using a central composite design for volatile carbonyl compounds determination in beers, Talanta 117 (2013) 523-531.

[19] S.L.C. Ferreira, R.E. Bruns, H.S. Ferreira, G.D. Matos, J.M. David, G.C. Brandão, E.G.P.d. Silva, L.A. Portugal, P.S.d. Reis, A.S. Souza, W.N.L.d. Santos, BoxBehnken design: an alternative for the optimization of analytical methods, Anal. Chim. Acta 597 (2007) 179-186.

[20] C.A. Ballus, A.D. Meinhart, F.A. de Souza Campos Jr., R.E. Bruns, H.T. Godoy, Doehlert design-desirability function multi-criteria optimal separation of 17 phenolic compounds from extra-virgin olive oil by capillary zone electrophoresis, Food Chem. 146 (2014) 558-568.

[21] S.L.C. Ferreira, R.E. Bruns, E.G.P.d. Silva, W.N.L.d. Santos, C.M. Quintella, J.M. David, J.B.d. Andrade, M.C. Breitkreitz, I.C.S.F. Jardim, B.B. Neto, Statistical designs and response surface techniques for the optimization of chromatographic systems, J. Chromatogr. A 1158 (2007) 2-14.

[22] L.V. Candioti, M.M.D. Zan, M.S. Cámara, H.C. Goicoechea, Experimental design and multiple response optimization. Using the desirability function in analytical methods development, Talanta 124 (2014) 123-138.

[23] T. Pérez-Palacios, C. Petisca, A. Melo, I.M. Ferreira, Quantification of furanic compounds in coated deep-fried products simulating normal preparation and consumption: optimisation of HS-SPME analytical conditions by response surface methodology, Food Chem. 135 (2012) 1337-1343.

[24] C.-W. Ye, J. Gao, C. Yang, X.-J. Liu, X.-J. Li, Development and application of an 
SPME/GC method for the determination of trace phthalates in beer using a calix[6]arene fiber, Anal. Chim. Acta 641 (2009) 64-74.

[25] Q.L. Ma, N. Hamid, A.E.D. Bekhit, J. Robertson, T.F. Law, Optimization of headspace solid phase microextraction (HS-SPME) for gas chromatography mass spectrometry (GC-MS) analysis of aroma compounds in cooked beef using response surface methodology, Microchem. J. 111 (2013) 16-24.

[26] A.C. Atkinson, A.N. Donev, Optimum Experimental Designs, Oxford University Press, Oxford, 1992.

[27] S.H. Munson-McGee, D- and G-optimal experimental designs for the partition coefficient in freeze concentration, J. Food Eng. 121 (2014) 80-86.

[28] R. Hoogerbrugge, A.M. Stolker, A. Barendregt, E. Hogendoorn, A systematic approach for optimisation of supercritical-fluid extraction of polycyclic aromatic hydrocarbons from earthworms, Anal. Bioanal. Chem. 377 (2003) $715-722$.

[29] I. García, L. Sarabia, M. Cruz Ortiz, J.M. Aldama, Usefulness of D-optimal designs and multicriteria optimization in laborious analytical procedures: application to the extraction of quinolones from eggs, J. Chromatogr. A 1085 (2005) 190-198.

[30] T. Shibamoto, Diacetyl: occurrence, analysis, and toxicity, J. Agric. Food Chem. 62 (2014) 4048-4053.

[31] K. Krogerus, B. Gibson, Influence of valine and other amino acids on total diacetyl and 2,3-pentanedione levels during fermentation of brewer's wort, Appl. Microbiol. Biot. 97 (2013) 6919-6930.

[32] B. Vanderhaegen, H. Neven, H. Verachtert, G. Derdelinckx, The chemistry of beer aging - a critical review, Food Chem. 95 (2006) 357-381.

[33] T. Inoue, A review of diacetyl control technology, Proc. Conv. Inst. Brew. 2 (1992) 76-79.

[34] C.W. Bamforth, M. Kanauchi, Enzymology of vicinal diketone reduction in Brewer's yeast, J. Inst. Brew. 110 (2004) 83-93.

[35] T. Brányik, A.A. Vicente, P. Dostálek, J.A. Teixeira, A review of flavour formation in continuous beer fermentations, J. Inst. Brew. 114 (2008) 3-13.

[36] A. De Stefano, L. Montanari, Minor components of beer: a review, Alcologia 8 (1996) 43-45.

[37] K. Krogerus, B.R. Gibson, 125th anniversary review: diacetyl and its control during brewery fermentation, J. Inst. Brew. 119 (2013) 86-97.

[38] P. Li, Y. Zhu, S. He, J. Fan, Q. Hu, Y. Cao, Development and validation of a highperformance liquid chromatography method for the determination of diacetyl in beer using 4-nitro-o-phenylenediamine as the derivatization reagent, J. Agric. Food Chem. 60 (2012) 3013-3019.

[39] M. Yamaguchi, J. Ishida, Z.X. Xuan, A. Nakamura, T. Yoshitake, Determination of glyoxal, methylglyoxal, diacethyl, and 2, 3-pentanedione in fermented foods by high-performance liquid chromatography with fluorescence detection, J. Liq. Chromatogr. 17 (1994) 203-211.

[40] G.A.F. Harrison, W.J. Byrne, E. Collins, The determination of diacetyl and 2,3pentanedione in beer head speace by gas chromatography, J. Inst. Brew. 71 (1965) 336-341.

[41] S. Landaud, P. Lieben, D. Picque, Quantitative analysis of diacetyl, pentanedione and their precursors during beer fermentation by an accurate GC/MS method, J. Inst. Brew. 104 (1998) 93-99.
[42] J.D. Pejin, O.S. Grujić, N.J. Marjanović, Đ.N. Vujić, S.D. Kocić-Tanackov, Application of GC/MS method using SPE columns for quantitative determination of diacetyl and 2,3-pentanedione during beer fermentation, J. Am. Soc. Brew. Chem. 64 (2006) 52-60.

[43] J.D. Pejin, O.S. Grujić, N.J. Marjanović, Đ.N. Vujić, S.D. Kocić-Tanackov, Determination of diacetyl and 2,3-pentanedione in beer by gc/ms using solid-phase extraction columns, APTEFF 33 (2002) 45-54.

[44] M. Povolo, G. Contarini, Comparison of solid-phase microextraction and purge-and-trap methods for the analysis of the volatile fraction of butter J. Chromatogr. A 985 (2003) 117-125.

[45] C. Condurso, A. Verzera, V. Romeo, M. Ziino, F. Conte, Solid-phase microextraction and gas chromatography mass spectrometry analysis of dairy product volatiles for the determination of shelf-life, Int. Dairy J. 18 (2008) $819-825$.

[46] A. Rivas-Cañedo, C. Juez-Ojeda, M. Nuñez, E. Fernández-García, Volatile compounds in ground beef subjected to high pressure processing: a comparison of dynamic headspace and solid-phase microextraction, Food Chem 124 (2011) 1201-1207.

[47] M. Bueno, J. Zapata, V. Ferreira, Simultaneous determination of free and bonded forms of odor-active carbonyls in wine using a headspace solid phase microextraction strategy, J. Chromatogr. A 1369 (2014) 33-42.

[48] H. Kataoka, H.L. Lord, J. Pawliszyn, Applications of solid-phase microextraction in food analysis, J. Chromatogr. A 880 (2000) 35-62.

[49] R.T. Johnson, D.C. Montgomery, B.A. Jones, An expository paper on optimal design, Qual. Eng. 23 (2011) 287-301.

[50] R.K. Meyer, C.J. Nachtsheim, The coordinate exchange algorithm for constructing exact optimal designs, Technometrics 37 (1995) 60-69.

[51] K. Danzar, L.A. Currie, Guidelines for calibration in analytical chemistry, Pure Appl. Chem. 70 (1998) 993-1014.

[52] B.K. Matuszewski, M.L. Constanzer, C.M. Chavez-Eng Strategies for the assessment of matrix effect in quantitative bioanalytical methods based on HPLC-MS/MS, Anal. Chem. 75 (2003) 3019-3030.

[53] I. Taverniers, M. De Loose, E. Van Bockstaele, Trends in quality in the analytical laboratory. II. Analytical method validation and quality assurance, Trend Anal. Chem. 23 (2004) 535-552.

[54] O. Ballesteros, A. Zafra, A. Navalón, J.L. Vílchez, Sensitive gas chromatographic-mass spectrometric method for the determination of phthalate esters, alkylphenols, bisphenol $\mathrm{A}$ and their chlorinated derivatives in wastewater samples, J. Chromatogr. A 1121 (2006) 154-162.

[55] P. Araujo, Key aspects of analytical method validation and linearity evaluation, J. Chromatogr. A 877 (2009) 2224-2234.

[56] M. Villar, M. Callejón, J.C. Jiménez, E. Alonso, A. Guiraúm, New rapid methods for determination of total LAS in sewage sludge by high performance liquid chromatography (HPLC) and capillary electrophoresis (CE), Anal. Chim. Acta 634 (2009) 267-271.

[57] S. Cantarero, A. Zafra-Gómez, O. Ballesteros, A. Navalón, J.L. Vílchez, C. Verge J.A. De Ferrer, Matrix effect study in the determination of linear alkylbenzene sulfonates in sewage sludge samples, Environ. Toxicol. Chem. 30 (2011) $813-818$. 